\title{
COR-Like Gene Is Involved in Induced-Expression Response to Multiple Abiotic Stresses in Grape Vine (Vitis amurensis) Tissues
}

\author{
Chang Dong*, Yang Qin, Bailin Wang, Huiling Lu, Lizhen Xiao, Ruihua Yang, Yu Wang, \\ Lixin Chen, Yixin Feng \\ Department of Horticulture, Heilongjiang Academy of Agricultural Science, Harbin, China \\ Email: ${ }^{\text {dongchanggy@126.com }}$
}

Received 6 March 2014; revised 15 May 2014; accepted 3 June 2014

Copyright (C) 2014 by authors and Scientific Research Publishing Inc.

This work is licensed under the Creative Commons Attribution International License (CC BY).

http://creativecommons.org/licenses/by/4.0/

(c) (i) Open Access

\begin{abstract}
Cold stress, which causes dehydration damage to plants, is one of the most common abiotic stresses that limit plant distributions and affect crop growth and development. To improve their cold tolerance, plants often upregulate the expression of some cold-related genes. In this study, a cold-regulated (COR) gene was isolated from Vitis amurensis and designated as VaCOR. RT-PCR analysis demonstrated that VaCOR was expressed at high levels in the roots, stems, leaves, and petioles under low temperature, but it was not detected under normal temperatures. Further analysis revealed that salinity and the application of exogenous abscisic acid and salicylic acid significantly induced VaCOR transcription, with apparent differences in its expression in different organs. The data also showed that COR gene expression was higher in cold-resistant wild $V$. amurensis than in cold-sensitive Vitis vinifera "Manicure Finger" under low temperature. These results suggest that the VaCOR gene in V. amurensis grapes is involved in multiple stresses and plays a central role in stress-induced and stress-tolerance.
\end{abstract}

\section{Keywords}

Vitis amurensis, COR, Stress, Expression

\section{Introduction}

Cold is one of the most common abiotic stresses that adversely affect crop growth and development. Previous

${ }^{*}$ Corresponding author.

How to cite this paper: Dong, C., et al. (2014) COR-Like Gene Is Involved in Induced-Expression Response to Multiple Abiotic Stresses in Grape Vine (Vitis amurensis) Tissues. Agricultural Sciences, 5, 604-610.

http://dx.doi.org/10.4236/as.2014.57063 
studies have indicated that plants increase their cold tolerance when exposed to nonfreezing temperatures through an adaptive process called cold acclimation, which is associated with a number of biochemical and physiologic changes [1]. These changes are correlated with changes in gene expression.

In the past decades, considerable effort has been directed at identifying cold-regulated (COR) genes in both dicotyledonous and monocotyledonous plant species. A previous study indicates that the Arabidopsis thaliana COR15a gene encodes a chloroplast polypeptide [2], and that COR15a overexpression significantly increases the cellular freezing tolerance of Arabidopsis [3]. Similarly, the barley COR14b gene, one of the best-characterized cold-regulated genes in cereals, is strictly regulated by cold [4]. Furthermore, barley accumulates more COR14b proteins in winter than in spring [5]. The wheat COR14b protein has also been identified and is located in the chloroplast stroma [6]. Therefore, the COR14b gene has been employed as a genetic tool for identifying components involved in the cold signaling pathway [7] [8]. In addition, a study reveals that the wheat COR15 gene improves the cellular freezing tolerance of tobacco plants [9]. A more recent study reveals that Camellia sinensis COR1 expression is up-regulated by cold, abscisic acid (ABA), and dehydration [10]. These findings indicate that the accumulation of COR proteins is related to plant cold resistance. Additionally, studies have indicated that the promoters of COR homolog contain dehydration responsive elements (DRE)/C-repeat (CRT) elements, and other stress responsive cis-elements called ABRE, MYB, and MBS [11] [12]. Overexpression of CRT/DRE binding factor (CBF) transcription factor induces or up-regulates COR homolog gene expression [12] [13].

Vitis amurensis is one of the most widely used species for freezing-resistant rootstock and winemaking in grape cultivation. In this study, we isolated the VaCOR gene from $V$. amurensis and compared the COR gene expression pattern in the leaves of cold-tolerant and cold-sensitive grape species under low temperatures. Furthermore, we analyzed the COR transcript patterns in the roots, stems, leaves, and petioles of $V$. amurensis under low temperature, high salinity, and abscisic acid (ABA) and salicylic acid (SA) treatments at different exposure times.

\section{Materials and Methods}

\subsection{Cloning and Bioinformatics Analysis of VaCOR}

VaCOR was cloned from single-stranded cDNA synthesized from the RNA of wild $V$. amurensis in vitro under cold stress conditions. Total RNA was extracted using a previously described method [14]. First-strand cDNA was synthesized according to the instructions included with the ReverTra Ace RT Kit (TOYOBO, Japan). The complete coding sequence was amplified using the primers CoF (5'-CCAGCTTCGGAGTTTAAG-3') and CoR (5'-CTGCATATAGAAAAACTCAAT-3'), which were designed based on the grape genome

(http://www.vitisgenome.it) according to the sequence of the rice COR gene (GenBank: AAT47009). The conserved protein domains were predicted using Motif Scan (http://scansite.mit.edu/motifscan_id.phtml). The amino acid sequences of COR in other species were obtained from the National Center of Biotechnology Information (NCBI, http://www.ncbi.nlm.nih.gov/) database by BLAST. Multiple sequence alignment of the COR conserved domains was performed using Clustal 1.83. Shading was conducted using DNAMAN software (Version 5.2.2, Lynnon Biosoft).

\subsection{Treatment of Materials}

Sixty-day-old in vitro samples of wild V. amurensis and Vitis vinifera "Manicure Finger" grown in Murashige and Skoog (MS) medium under a $16 \mathrm{~h} \mathrm{light} / 8 \mathrm{~h}$ dark regime at $25^{\circ} \mathrm{C}$ were subjected to the following treatments for stress-responsive gene expression: cold stress at $4^{\circ} \mathrm{C}$, salinity stress under $200 \mathrm{mM} \mathrm{NaCl}$, and hormonal treatments with $10 \mu \mathrm{M}$ ABA or $5 \mu \mathrm{M}$ SA at different exposure times.

\subsection{Expression Pattern of COR}

Total RNA and first-strand cDNA were obtained as described above. Total RNA from different materials were digested with RNase-free DNase I (TaKaRa, Japan) to remove genomic DNA contamination. Transcription levels were determined on an ABI PRISM 7300 Real-time PCR System (Applied Biosystems, Foster City, CA, USA). For each reaction, 20 ng of cDNA was used with SYBR Premix Ex Taq mix (TaKaRa), including the primers Co1 (5'-ACATTCCACCGACCCTG-3') and Co2 (5'-TCCTCGTCCAAAGCATAGA-3') for the VaCOR gene. A positive control was provided through parallel analysis using the grape malate dehydrogenase 
gene as the internal reference. Amplification data from the roots at $0.5 \mathrm{~h}$ and $4{ }^{\circ} \mathrm{C}$ were used for comparison. Relative expression was calculated relative to a calibrator using the formula $2^{-\Delta \Delta \mathrm{Ct}}$. Two independent replicates were performed per experiment.

\section{Results}

\subsection{Isolation and Characterization of the VaCOR Gene}

Based on the sequence analysis, a 691 bp cDNA sequence containing a 453 bp open reading frame (ORF) was obtained. The ORF encoded a deduced 151-amino acid protein with a predicted molecular mass of $517 \mathrm{kDa}$ and a theoretical isoelectric point of 7.0. Protein sequence alignment showed that VaCOR shares $86 \%$ identity with VvCOR, 65\% identity with PtCOR, and 55\% identity with OsCOR (Figure 1), and was therefore designated as VaCOR (GenBank Accession No. HM231146). However, homology searches failed to identify any apparent homologous COR sequences in Arabidopsis. Amino acid sequence analysis revealed that VaCOR displays a number of dehydrin features and that it belongs to the Phosphatidyl ethanolamine-binding protein superfamily of Glycine- and Leucine-rich proteins. In addition, several conserved domains LPEGFSGKE were found in the protein. This feature, however, is not found in other dehydrin or COR proteins in Arabidopsis.

\subsection{Expression of COR in Grape Species under Low-Temperature Stress}

Expression patterns of the COR gene in leaves of different grape species were analyzed by RT-PCR and qRTPCR. The results showed that VvCOR is mainly expressed in untreated plants and those in the early stages of low temperature exposure, but its mRNA was found at low levels within 1 day to 2 days under low temperature (Figure 2(a)). However, VaCOR mRNA was not found in the untreated controls, which was confirmed through qRT-PCR. The VaCOR expression levels were significantly increased under low temperature (Figure 2(a) and Figure 2(b)). The expression levels peaked within $4 \mathrm{~h}$ to $10 \mathrm{~h}$ under cold treatment, and subsequently declined. By contrast, the VvCOR expression levels dropped after $4 \mathrm{~h}$ cold treatment. These results indicate that low temperature induced VaCOR expression in the leaves, whereas low temperature down-regulated VvCOR expression.

\subsection{Expression Patterns of VaCOR at Low Temperature}

Accumulation of each transcript in the roots, stems, leaves, and petioles during cold treatment was analyzed through quantitative RT-PCR to determine further the acquisition mechanisms of cold acclimation in relation to VaCOR gene expression. The results show that low temperature induced VaCOR accumulation in the roots, stems, leaves, and petioles and exhibited different expression levels in different organs (Figure 3). During the chilling period, the transcription VaCOR levels in all organs fluctuated and peaked after $8 \mathrm{~h}$ cold treatment. In

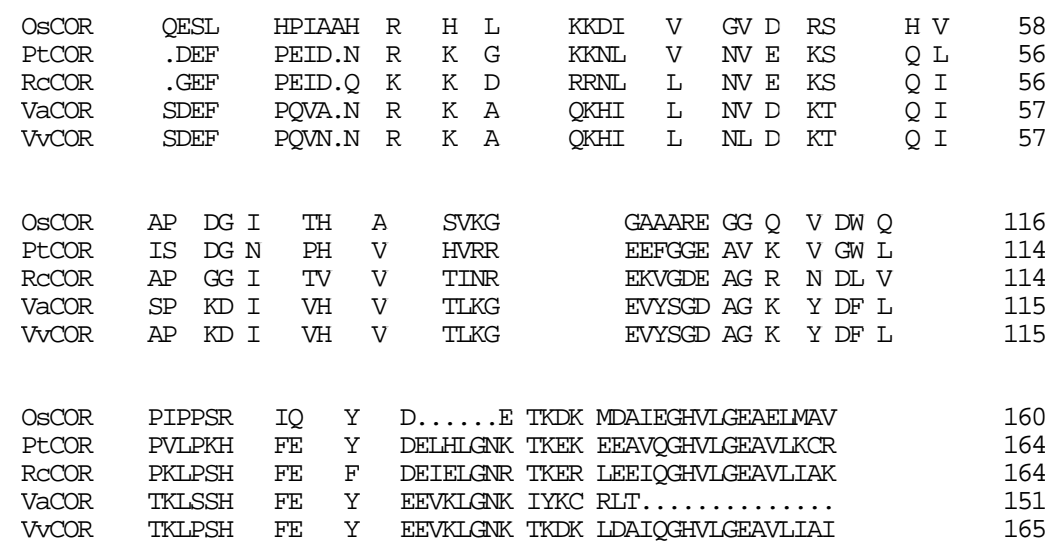

Figure 1. Comparison of the amino acid sequences of COR and its homolog. The black areas denote the highly conserved amino acid sequences. Accession numbers of the known proteins in GenBank: OsCOR, AAT47009; PtCOR, XP_002323004; RcCOR, XP_002530493; VaCOR, HM231146; VvCOR, XP_002271217. 


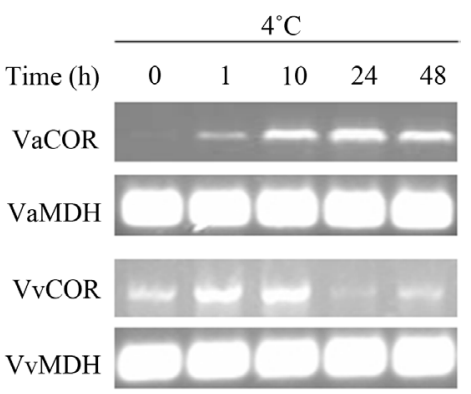

(a)

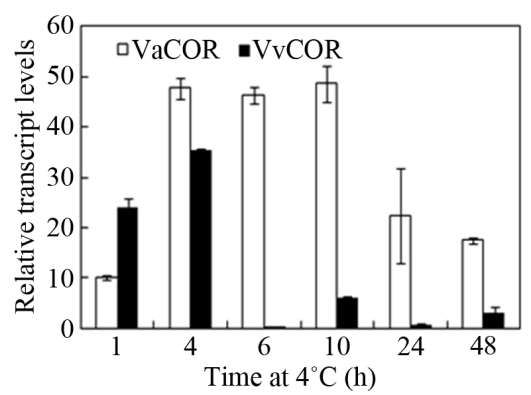

(b)

Figure 2. Expression analysis of the COR gene in grape species under low temperature. (a) RT-PCR analysis of $V$. amurensis (Va) and $V$. vinifera $(\mathrm{Vv})$ under normal conditions and $4^{\circ} \mathrm{C}$; (b) qRT-PCR analysis in leaves of different grape species at $-4^{\circ} \mathrm{C}$. Bars indicate the standard deviation.

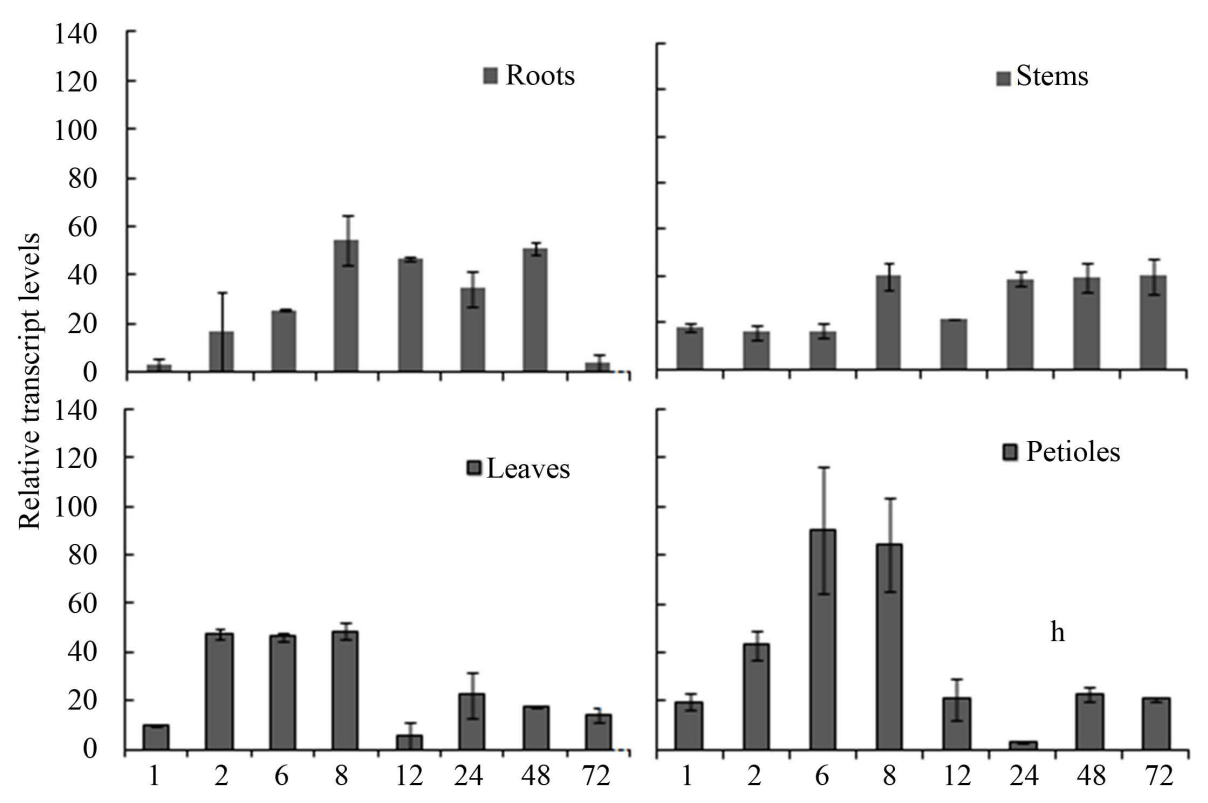

Figure 3. Expression patterns of the VaCOR gene in different tissues at $4^{\circ} \mathrm{C}$. Bars indicate the standard deviation. Bars indicate the standard deviation.

the leaves, $\mathrm{VaCOR}$ transcript accumulation was slightly increased during the early stage of treatment and peaked from $2 \mathrm{~h}$ to $8 \mathrm{~h}$, and then declined. Similar results were observed in the petioles with the leaves, but the peak occurred after $6 \mathrm{~h}$ to $8 \mathrm{~h}$. The VaCOR transcription levels in the stems peaked after $8 \mathrm{~h}$ and maintained a high transcription level. By contrast, the mRNA expression in the roots was slightly increased after $1 \mathrm{~h}$, gradually reached its peak after $8 \mathrm{~h}$, remained almost constant until after $48 \mathrm{~h}$ of treatment, and then declined after $72 \mathrm{~h}$.

\subsection{Expression Patterns of VaCOR under High Salinity}

In general, the stress responses of genes have multiplicity and can be triggered by different kinds of stress in different organs. We analyzed the expression of the VaCOR gene under high salinity to obtain more information regarding its expression under different kinds of stress. The results show that salinity induced VaCOR expression throughout the treatment period (Figure 4). The VaCOR transcript accumulation in all organs displayed a single peak. However, different organs displayed distinct gene expression levels during the treatment period. In the roots, the VaCOR expression sharply peaked during the early stage of treatment, and subsequently declined to low levels after $72 \mathrm{~h}$. By contrast, the peak transcript accumulation in the leaves occurred after $10 \mathrm{~h}$, whereas that in the stems and petioles occurred after $12 \mathrm{~h}$. 


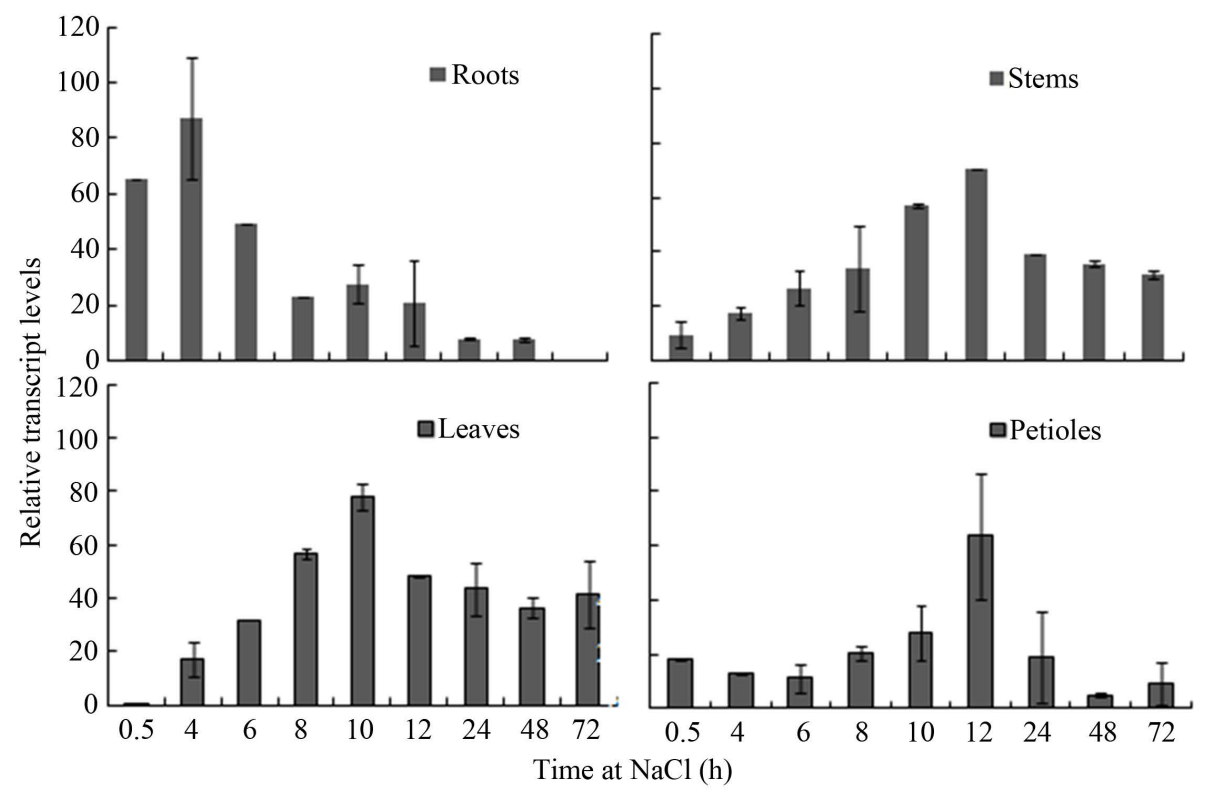

Figure 4. Expression patterns of the VaCOR gene in different organs under $\mathrm{NaCl}$ treatment. Bars indicate the standard deviation.

\subsection{ABA and SA Activated VaCOR}

$\mathrm{ABA}$ and $\mathrm{SA}$ are important exogenous hormones involved in physiologic and genetic regulation during stress. Therefore, we hypothesized that ABA and SA affect the VaCOR expression patterns. The results show that exogenous ABA and SA treatments induced the VaCOR gene (Figure 5). However, the different organs exhibited significant differences in VaCOR gene expression under the ABA and SA treatments. Under the ABA treatment, the transcript accumulation in the roots was greatly increased, reaching its peak within $1 \mathrm{~h}$, and then declining to almost none after $72 \mathrm{~h}$. By contrast, those in the stems and the leaves were activated by ABA application, but the accumulation was not significant, especially in the leaves. Under the SA treatment, the VaCOR expression patterns in all organs decreased after $10 \mathrm{~h}$ of treatment. The VaCOR expression in the roots and stems were significantly induced and fluctuated, whereas that in the leaves was not significant under the ABA treatment.

\section{Discussion}

In this study, a novel cold-regulated gene was isolated from V. amurensis and designated as VaCOR because it encodes a protein that is highly homologous to other COR genes in other plants. However, our analysis indicated that no homologous cold-regulated protein occur in Arabidopsis (data not shown). Therefore, the isolated VaCOR may represent a novel COR proteins involved in cold acclimation in $V$. amurensis. Its most typical feature is the conserved domain in its central region, followed by a cluster of charged amino acid residues (Figure 1).

The data revealed that COR transcripts are significantly induced or up-regulated in Vitis species upon exposure to low temperatures (Figure 2). However, the COR gene expression levels were distinctly higher in coldresistant $V$. amurensis than in moderately cold-sensitive $V$. vinifera under low temperature stress (Figure 2). Furthermore, the transcript accumulation in $V$. vinifera sharply increased during the early stages of treatment (1 h), and then declined to low levels, whereas the peak accumulation in $V$. amurensis occurred during the midstage of treatment. The different expression patterns of grape COR genes indicated that the VaCOR gene improves the cold tolerance of plants.

Similar to most COR genes in other plants, the VaCOR gene responded to cold in different organs. Our results show that the COR transcripts were abundant in different tissues (roots, stems, leaves, and petioles) and lasted for long periods under low temperatures. Furthermore, the grape COR transcripts that accumulated in the petioles were higher and displayed an obvious single peak during the whole low temperature treatment (Figure 3). Furthermore, the expression in the leaves, stems, and roots were induced under cold treatment. Liang et al. [15] indicated that Brassica COR gene homologues are induced by cold, dehydration, and ABA stresses. In this 


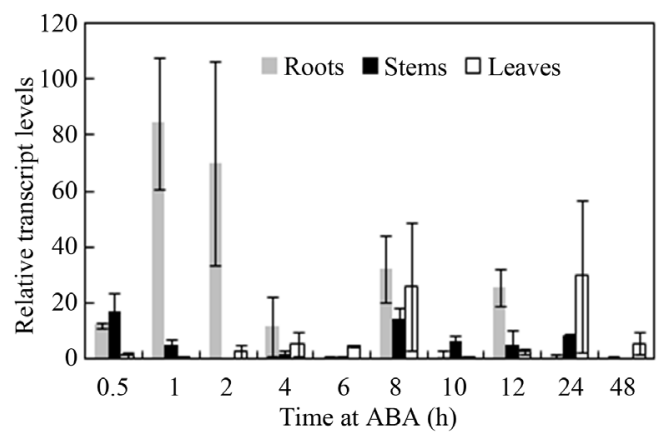

(a)

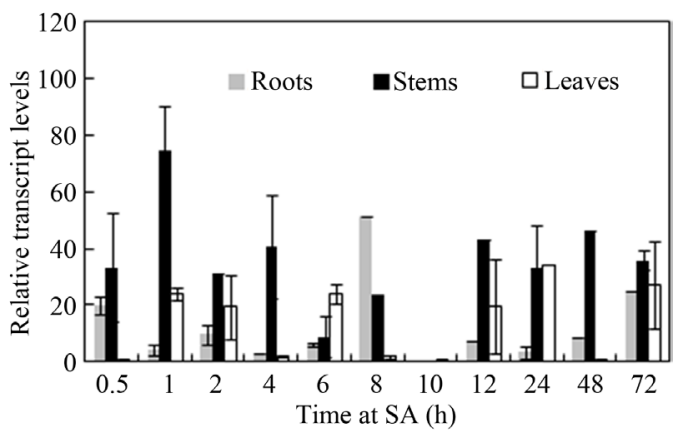

(b)

Figure 5. Expression patterns of the VaCOR gene in different organs under ABA and SA treatments. Bars indicate the standard deviation.

study, $V$. amurensis COR was induced by high salinity, and displayed different accumulation levels in organs. These results indicate that different organs respond differently to stress signals.

Several studies have demonstrated that COR is regulated by abiotic stresses, such as ABA [15] [16]. In the present study, VaCOR expression was triggered by exogenous ABA and SA (Figure 5). ABA and SA, signal molecules that affect growth and development of plants, are involved in plant responses to several environmental stress factors and reportedly act as important activators in the cold CBF pathway [17]. Furthermore, SAresponsive elements such as TCA-element and ABA-responsive elements including ABRE in the 5'-flanking region of key CBF genes have been found and reported previously [13] [18], which suggests that ABA and SA are effective regulators for stimulating gene expression to cope with cold and other abiotic stresses. This study demonstrated that ABA and SA activated the COR gene in V. amurensis in different organs, and displayed different accumulation patterns in the roots, stems, and petioles (Figure 5). Accumulation in roots and stems is relatively higher than that in the leaves, and this pattern may be related to differences in cold resistance in different organs. Further studies are needed to verify these findings.

In summary, cDNA clones were identified and stress-responsive characterization in tissues of VaCOR from $V$. amurensis under low temperatures and salinity. The application of ABA and SA was conducted. The information obtained from this study provides a foundation from which to explore the gene regulatory networks under stresses, determine the signal diversities of organs upon exposure to stress in Vitis, and investigate other molecular or cell biological approaches.

\section{Acknowledgements}

This work was supported by the National Technology System for Grape Industry (No. CARS-30-25).

\section{References}

[1] Thomashow, M.F. (1999) Plant Cold Acclimation Freezing Tolerance Genes and Regulatory Mechanisms. Plant Physiology, 50, 571-599.

[2] Baker, S.S., Wilhelm, K.S. and Thomashow, M.F. (1994) The 5'-Region of Arabidopsis thaliana cor15a Has Cis-Acting Elements That Confer Cold-, Drought- and ABA-Regulated Gene Expression. Plant Molecular Biology, 24, 701-713. http://dx.doi.org/10.1007/BF00029852

[3] Artus, N.N., et al. (1996) Constitutive Expression of the Cold Regulated Arabidopsis thaliana COR15a Gene Affects Both Chloroplast and Protoplast Freezing Tolerance. Proceedings of the National Academy of Sciences of the United States of America, 93, 13404-13409. http://dx.doi.org/10.1073/pnas.93.23.13404

[4] Cattivelli, L. and Bartels, D. (1990) Molecular Cloning and Characterization of Cold-Regulated Genes in Barley. Plant Physiology, 93, 1504-1510. http://dx.doi.org/10.1104/pp.93.4.1504

[5] Giorni, E., et al. (1999) Cold-Regulated Gene Expression during Winter in Frost Tolerant and Frost Susceptible Barley Cultivars Grown under Field Conditions. Euphytica, 160, 5873-7877.

[6] Crosatti, C., Polverino, L.P., Bassi, R. and Cattivelli, L. (1999) The Interaction between Cold and Light Controls the Expression of the Cold-Regulated Barley Gene Cor14b and the Accumulation of the Corresponding Protein. Plant Physiology, 119, 671-680. http://dx.doi.org/10.1104/pp.119.2.671 
[7] Crosatti, C., et al. (2003) Genetic Analysis of the Expression of the Cold Regulated Gene cor14b: A Way toward the Identification of Components of the Cold Response Signal Transduction in Triticeae. Canadian Journal of Botany, 81, 1162-1167. http://dx.doi.org/10.1139/b03-114

[8] Rapacz, M., Wolanin, B., Hura, K. and Tyrka, M. (2008) The Effects of Cold Acclimation on Photosynthetic Apparatus and the Expression of COR14b in Four Genotypes of Barley (Hordeum Vulgare) Contrating in Their Tolerance to Freezing and High-Light Treatment in Cold Conditions. Annals of Botany, 101, 689-699. http://dx.doi.org/10.1093/aob/mcn008

[9] Shimamura, C., Ohno, R., Nakamura, C. and Takumi, S. (2006) Improvement of Freezing Tolerance in Tobacco Plants Expressing a Cold-Responsive and Chloroplast-Targeting Protein WCOR15 of Wheat. Journal of Plant Physiology, 1, 213-219. http://dx.doi.org/10.1016/j.jplph.2005.06.008

[10] Li, X.W., et al. (2010) A novel Cold-Regulated Gene from Camellia sinensis, CsCOR1, Enhances Salt- and Dehydration-Tolerance in Tobacco. Biochemical and Biophysical Research Communications, 394, 354-359. http://dx.doi.org/10.1016/j.bbrc.2010.03.011

[11] Yamaguchi-Shinozaki, K. and Shinozaki, K. (1994) A Novel Cis-Acting Element in an Arabidopsis Gene Is Involved in Responsiveness to Drought, Low-Temperature, or High-Salt Stress. The Plant Cell, 6, 251-264. http://dx.doi.org/10.1105/tpc.6.2.251

[12] Stockinger, E.J., Gilmour, S.J. and Thomashow, M.F. (1997) Arabidopsis thaliana CBF1 Encodes an AP2 Domain-Containing Transcriptional Activator That Binds to the C-repeat/DRE, a Cis-Acting DNA Regulatory Element That Stimulates Transcription in Response to Low Temperature and Water Deficit. Proceedings of the National Academy of Sciences of the United States of America, 94, 1035-1040. http://dx.doi.org/10.1073/pnas.94.3.1035

[13] Dong, C., et al. (2013) VaCBF1 from Vitis amurensis Associated with Cold Acclimation and Cold Tolerance. Acta Physiologiae Plantarum, 6, 1-8.

[14] Lagonigro, M.S., et al. (2004) CTAB-Urea Method Purifies RNA from Melanin for cDNA Microarray Analysis. Pigment Cell Research, 17, 312-315. http://dx.doi.org/10.1111/j.1600-0749.2004.00155.x

[15] Liang, C., et al. (2011) A Novel Cold-Regulated Gene, COR25, of Brassica napus Is Involved in Plant Response an Tolerance to Cold Stress. Plant Cell Reports, 30, 463-471. http://dx.doi.org/10.1007/s00299-010-0952-3

[16] Steponkus, P.L., Uemura, M., Joseph, R.A., Gilmour, S.J. and Thomashow, M.F. (1998) Mode of Action of the COR15a Gene on the Freezing Tolerance of Arabidopsis thaliana. Proceedings of the National Academy of Sciences of the United States of America, 95, 14570-14575. http://dx.doi.org/10.1073/pnas.95.24.14570

[17] Ruelland, E., Vaultier, M.N. and Hurry, A and Za, V. (2009) Cold Signalling and Cold Acclimation in Plants. Advances in Botanical Research, 49, 35-150. http://dx.doi.org/10.1016/S0065-2296(08)00602-2

[18] Dong, C., et al. (2013) Isolation and Expression Analysis of CBF4 from Vitis amurensis Associated with Stress. Agricultural Sciences, 4, 224-229. http://dx.doi.org/10.4236/as.2013.45032 